\title{
Aspekte zur Diversität der Polychaeta (Annelida) unter besonderer Berücksichtigung der Publikationen von Volker Storch nebst der Beschreibung einer neuen Art der Ampharetidae
}

\author{
Thomas Wehe
}

Eingegangen: 7. Januar 2009/Akzeptiert: 23. Februar 2009/Online veröffentlicht: 21. März 2009

(C) Springer-Verlag 2009

Zusammenfassung Hintergrund und Ziel Unter dem Begriff Biodiversität versteht man heute sowohl die eigentliche Artenvielfalt als auch die biologische Vielfalt auf supra- und subspezifischen Ebenen. Der Artikel beleuchtet am Beispiel der Polychaeta Aspekte der Vielfalt in diesem Sinne. Dabei nimmt er besonderen Bezug auf das wissenschaftliche Werk zu Borstenwürmern von Volker Storch. Aus Anlass seines 65. Geburtstages wird die Beschreibung einer neuen Art Volker Storch gewidmet.

Ergebnisse In mehr als vierzig Jahren systematisch-faunistischer, histologischer, morphologischer und ultrastruktureller Forschung hat Volker Storch, neben unzähligen weiteren Tiergruppen, wesentlich zu unseren Kenntnissen über Polychaeta beigetragen. Mehrere Arten wurden von ihm oder seinen Mitarbeitern neu entdeckt und beschrieben. Morphologisch und ultrastrukturell wurden besonders intensiv die Muskulatur, das Integument, der Verdauungstrakt, Sinnesrezeptoren und Kiemen untersucht. Da von grundlegender Bedeutung, finden sich die Resultate dieser Arbeiten in den einschlägigen Handbuchreihen und Lehrbüchern wieder.

Neben der Darstellung der Biodiversität anhand der Arbeiten von Volker Storch wird eine neue Art beschrieben, Samytha storchi Reuscher \& Wehe nov. spec. Die neue Art stammt aus dem nördlichen Golf von Akaba, Rotes Meer, Jordanien.

Diskussion und Schlussfolgerungen Die Zusammenschau der Veröffentlichungen von Volker Storch und weiterer im

Herrn Prof. Dr. Dr. h.c. Volker Storch gewidmet, anlässlich seines 65. Geburtstages.

Herausgeber: Henner Hollert · Thomas Braunbeck

T. Wehe $(\varangle)$

Universität Heidelberg, Zoologisches Institut,

Im Neuenheimer Feld 230, 69120 Heidelberg, Deutschland

E-Mail: thomas.wehe@yahoo.de
Text erwähnter Literatur zeigt beispielhaft, wie unser Wissen über die Vielfalt der Polychaeta in den letzten Jahrzehnten stetig zugenommen hat. Es wird aber auch deutlich, dass die Diversität dieser Tiere in all ihren Facetten bei weitem noch nicht erfasst ist. Bei etwa 13.000 bekannten Arten sind unsere Kenntnisse der Morphologie erwartungsgemäß nur exemplarischer Natur. Insbesondere was die Artenvielfalt und Phylogenie anbelangt offenbaren die fortlaufende Beschreibung neuer Arten und wechselnde Verwandtschaftshypothesen unsere Wissenslücken.

Empfehlungen und Perspektiven Zum Verständnis der Biodiversität der Polychaeta, deren Morphologie, Biologie und Phylogenie, bedarf es weiterer intensiver Forschungen. Dies erscheint umso mehr geboten vor dem Hintergrund der fortschreitenden Biodiversitätskrise.

Schlüsselwörter Ampharetidae · Biodiversität · Borstenwürmer $\cdot$ Morphologie $\cdot$ Polychaeta $\cdot$ Prof. Volker Storch · Rotes Meer · Samytha storchi · Systematik $\cdot$ Ultrastruktur

Aspects of the diversity of Polychaeta (Annelida) with special consideration of the publications by Volker Storch, and the description of a new species of Ampharetidae

Abstract Background, aim, and scope Nowadays, the term "biodiversity" stands not only for the diversity of species, but also for the diversity found on supra- and subspecies level. The article highlights some aspects of polychaete diversity in this broad meaning and thereby especially considers the scientific work on polychaetes by Volker Storch. On the occasion of his 65th birthday, a new species is named for Volker Storch. 
Results In more than forty years of systematical, faunistical, histological, morphological, and ultrastructural research, Volker Storch has been contributing tremendously to our knowledge of Polychaeta, not to name all the other animal groups. Several species have been newly described by him and his collaborators. His studies focused on the morphology and ultrastructure of the musculature, integument, alimentary tract, sensory cells, and gills. Of basic importance, the results of this research are considered in renowned handbook series and text books.

Besides the account on biodiversity on the basis of Volker Storch's publications, a new species is described, Samytha storchi Reuscher \& Wehe nov. spec. The new species was found in the northern Gulf of Aqaba, Red Sea, Jordan.

Discussion and conclusions The synopsis of Volker Storch's publications and further literature cited in the text exemplifies our increasing knowledge on the diversity of polychaetes over the last decades. However, it becomes also evident that the biodiversity of these animals in all its facets is, by far, not yet assessed. Considering about 13.000 known species, our knowledge of morphology is only of exemplary nature. Especially as regards species diversity and phylogeny, the ongoing description of new species and changing phylogenies disclose our gaps of knowledge.

Recommendations and perspectives For a better understanding of the biodiversity of Polychaeta, their morphology, biology and phylogeny, further research efforts are needed. This appears to be even more important given the ongoing biodiversity crises.

Keywords Ampharetidae · Biodiversity · Bristle worms · Morphology · Polychaeta · Prof. Volker Storch · Red Sea · Samytha storchi $\cdot$ Systematics $\cdot$ Ultrastructure

\section{Artendiversität}

Niemand kann heute sagen, wie viele Tierarten auf der Erde tatsächlich vorkommen. Die Anzahl wissenschaftlich beschriebener Arten wird gegenwärtig mit etwa 1,8 Mio. angegeben. Über die darüber hinaus existierenden Arten gibt es nur stark variierende Schätzungen. Hierüber wurde an anderer Stelle in dieser Zeitschrift schon berichtet (Storch und Wehe 2007). Bis heute sind nur etwa 250.000 Meerestiere beschrieben worden (Bouchet 2006), Schätzungen zum tatsächlichen Umfang der marinen Fauna reichen aber bis in die Millionen (Grassle und Maciolek 1992; Lambshead und Boucher 2003). Unter den zahlreichen im Meer lebenden Wirbellosen sind es vor allem Krebstiere (Crustacea), Schnecken (Gastropoda), Muscheln (Bivalvia) und die zu den Annelida gehörenden Polychaeta oder Borstenwürmer, die das Gros der Arten stellen. Von letzteren sind nach verbreiteter Angabe weltweit etwa 13.000 Arten beschrieben worden (Hartmann-Schröder 1993; Hutchings und Fauchald 2000), eine Existenz von 25.000 bis zu 30.000 Arten wird aber vermutet (Snelgrove et al. 1997).

Beeindruckender noch als die Artendiversität sind die schieren Individuenzahlen, in denen Borstenwürmer in bestimmten Bereichen auftreten können. Ein paar Zahlen mögen dies verdeutlichen: in der Gezeitenzone, dem Eulitoral, leben bis zu 30.000 Individuen pro $\mathrm{m}^{2}$ (Storch und Welsch 2004); bis zu 4.000 Individuen allein des Seeringelwurmes Nereis diversicolor können in einem Quadratmeter Wattenmeerboden der Nordsee vorkommen (Hartmann-Schröder 1983); auf einer Sedimentfläche von nur $10 \mathrm{~m}^{2}$ in der Bass Strait wurden 200 verschiedene Arten gefunden (Coleman et al. 1997); nach Vine (1986) befanden sich in einem 4,7 kg schweren Korallenblock aus dem Roten Meer 1441 Individuen aus 103 verschiedenen Arten. Borstenwürmer sind neben Muscheln die dominierende Gruppe in Weichböden und stellen dort zusammen etwa $80 \%$ der benthonischen Lebensgemeinschaft (Sanders 1968). Anhand dieser Daten wird deutlich, welche außerordentliche ökologische Bedeutung Borstenwürmern zukommt, insbesondere mit Blick auf die Remineralisierung organischer Substanz - etwa $3 \mathrm{t}$ Substrat können jährlich von 30.000 Würmern pro $\mathrm{m}^{2}$ durch den Darm transportiert werden (Storch und Welsch 2004) -, der Bioturbation des Meeresbodens sowie in der Funktion als Nahrungsquelle anderer Organismen. Untersuchungen haben ergeben, dass der Mageninhalt ein- bis zweijähriger Schollen zu über $40 \%$ aus Schwanzstücken des Wattwurms Arenicola marina besteht, wobei sich die Würmer bis zu 30mal während ihrer Lebensspanne verbeißen lassen können und damit im wahrsten Wortsinn eine regenerative Energiequelle darstellen. Es wurde errechnet, dass in Siedlungen des Wattwurms bis zu 4.000t Sand pro Hektar bewegt werden können, was einer einheitlichen Schicht von $32 \mathrm{~cm}$ Dicke entsprechen würde (Kundy 1990). Trotz gleichen Grundbauplans unterscheiden sich die einzelnen Arten zum Teil erheblich voneinander. Die Größenspanne reicht von wenigen Millimetern bis hin zu Metern, die Tiere können typische wurmförmige Gestalt haben, dabei mehr oder weniger vagil sein, sie können aber auch Körperformen vorweisen, die nicht ohne Weiteres an einen Wurm erinnern. Vielfach ist der Körper in einer Wohnröhre verborgen und nur eine Tentakelkrone ragt aus dieser hervor. Auch die innere Anatomie ist sehr verschieden. $\mathrm{Zu}$ der körperlichen Vielgestaltigkeit kommt häufig noch eine farbenfrohe Zeichnung hinzu.

Borstenwürmer waren immer beliebte Studienobjekte, kaum einer der großen Zoologen, der ihnen nicht eine Arbeit gewidmet hätte. Für den Anfänger eignet sich kaum ein Tier besser als ein Ringelwurm zum Verständnis des Coeloms, und will man sich über die diversen Reproduktions- und Vermehrungsweisen im Tierreich informieren, so bieten die Borstenwürmer allein einen großen Überblick über vorhandene Modi. Bemerkenswerte Beispiele bieten 
der Palolowurm (Palola viridis, Eunicidae) oder Vertreter der Syllidae, wie die Gattung Autolytus. Bei ersterer, im Pazifik vorkommender, Art ist die Fortpflanzung durch die Mondphasen synchronisiert. Nur die Hinterenden der Männchen und Weibchen, prall gefüllt mit Geschlechtsprodukten, schwärmen im dritten Mondviertel im Oktober oder November. Die Körper werden durch die Brandung zerschlagen und es kommt zur Befruchtung der Gameten im freien Wasser. Bei Autolytus liegt ein Generationswechsel vor, wobei ungeschlechtlich erzeugte Individuen durch das Knospen von Tierketten entstehen.

\section{Die Erforschung der Borstenwürmer durch Volker Storch}

\subsection{Faunistisch-systematische Untersuchungen}

Die Artendiversität und die Vielfalt der Formen und Lebensäußerungen der Borstenwürmer haben auch das Interesse von Volker Storch vor etwas mehr als 40 Jahren geweckt, und die Beschäftigung mit diesen Tieren hat ihn seine wissenschaftliche Karriere hindurch begleitet. Von seinen über 250 Originalarbeiten zum gesamten Tierreich haben etwas weniger als ein Zehntel Borstenwürmer zum Thema. Zusammenfassende Artikel sind in einschlägigen Monographiereihen erschienen, entsprechende Kapitel finden sich in den eigenen Lehrbüchern. Dazu kommen durch ihn angeregte weitere Arbeiten von Kandidaten und Mitarbeitern. Die besondere Liebe zu den Borstenwürmern zeigt sich in dem bereits 1971 erschienenen Band ,Meeresborstenwürmer' der Neuen Brehm-Bücherei (Storch 1971).

Selbst auf Anregung seines Lehrers Prof. Adolf Remane zur Beschäftigung mit Polychaeten gebracht, beginnt die wissenschaftliche Laufbahn gleich mit einer Reihe von Publikationen zu diesen Tieren. Im Blickpunkt stehen hierbei zunächst faunistische Arbeiten. Remane und Schulz (1964) beschrieben zum ersten Mal einen von ihnen bei einem Forschungsaufenthalt am Roten Meer neu entdeckten Kleinstlebensraum. Im flachen Eulitoral, d.h. im Bereich des Riffdaches, gibt es dichte Bewüchse der buschig wachsenden Rotalge Digenia simplex. Die Algen sind über und über bedeckt und inkrustiert von Sand- und Kalkpartikeln und bieten so einen abwechslungsreichen Lebensraum für allerlei Kleinstorganismen, auch Borstenwürmer. Aus dieser nach Remane und Schulz (1964) genannten "Sandalgen-Zone“ werden zwei neue Arten beschrieben, der Syllidae Exogone remanei und der Spionidae Aonides nodosetosa (siehe Storch 1966). Es folgt die Beschreibung weiterer, von Volker Storch selbst im Roten Meer gesammelter Arten: Pisione reducta (Pisionidae), Syllis (Typosyllis) magnipectinis (Syllidae) und Paraonis (Paraonides) harpagonea (Paraonidae) (heute: Cirrophorus harpagoneus) sowie die Hesio- niden Gyptis ophiocomae und Podarke pugettensis spinapandens (heute zur Gattung Ophiodromus gestellt) (Storch 1967a, Storch und Niggemann 1967). Weitere Arten wurden entdeckt und zum ersten Mal im Roten Meer nachgewiesen. Die Erforschung von Borstenwürmern des Roten Meeres hat eine lange Geschichte in Deutschland. So war es Grube (1868, 1869), der fast genau 100 Jahre zuvor Polychaeten des Roten Meeres beschrieben hatte, nach den ersten von Napoleons Ägyptenfeldzug stammenden und von Savigny (1818, 1822) beschriebenen Arten (siehe auch Wehe et al. 2006).

Die Arbeit über Hesioniden ist nicht rein systematischer Natur, sondern auch klassische Naturbeobachtung und betont die Lebensweise der Tiere. Bei den beiden neuen Arten handelt es sich nämlich um kommensalisch lebende Formen, ein Phänomen, welches unter Borstenwürmern weit verbreitet ist. Viele Arten leben vergesellschaftet mit anderen marinen Wirbellosen und profitieren von deren Behausung oder Nahrungsresten, ohne ihnen dabei zu schaden. Auffällig ist hierbei das häufige Vorkommen auf bzw. mit Echinodermaten. Von 292 kommensalisch lebenden Borstenwurmarten leben $36 \%$ mit einer der fünf Klassen der Echinodermaten vergesellschaftet und von ca. 570 bekannten ,Wirtsarten' gehören ebenfalls 36\% zu den Stachelhäutern (Martin und Britayev 1998). Kommensalen finden sich am häufigsten unter den Hesionidae und Polynoidae, die letzteren sind auch als Schuppenwürmer bekannt. Die Beziehungen zwischen Kommensalen und Wirt sind meist artspezifisch. Die Untersuchungen von V. Storch an Podarke pugettensis spinapandens haben gezeigt, dass ihr Wirt, der irreguläre Seeigel Clypeaster humilis, ausschließlich diese Art akzeptiert, wohingegen andere Arten von den Pedicellarien und Stacheln des Seeigels bekämpft und in ihrer Fortbewegung behindert werden. Gyptis ophiocomae wiederum zeigt eine Zeichnung, die der seines Wirtes - dem Schlangenstern Ophicoma scolopendrina - täuschend ähnlich ist, so dass die Tiere auf ihrem Wirt nur sehr schwer auszumachen sind (Storch und Niggemann 1967).

Einer der häufigsten Schuppenwürmer in Korallenriffen des Roten Meeres (Wehe 2006) und bereits von Savigny (1818, 1822) beschrieben, ist Iphione muricata. Ebenfalls im Roten Meer kommt die nahe verwandte Art I. ovata vor (Abb. 1). Äußerlich ähneln diese Tiere den zu den Mollusken gehörenden Chitonen oder Käferschnecken (Polyplacophora). Diese Auffälligkeit veranlasste Storch (1967b), die Lebensweise von Iphione genauer zu studieren. Käferschnecken tragen auf ihrem Rücken acht in Reihe liegende Kalkplatten, die den Tieren bei oberflächlicher Betrachtung ein segmentiertes Erscheinen verleihen. Der Körper ist in der Regel breit, oval und abgeflacht. Sie besitzen ein umfangreiches und differenziert ausgeprägtes Muskelsystem, das es ihnen ermöglicht, sich fest an den Untergrund zu haften; man kann sich die Tiere gewissermaßen wie einen Saugnapf 


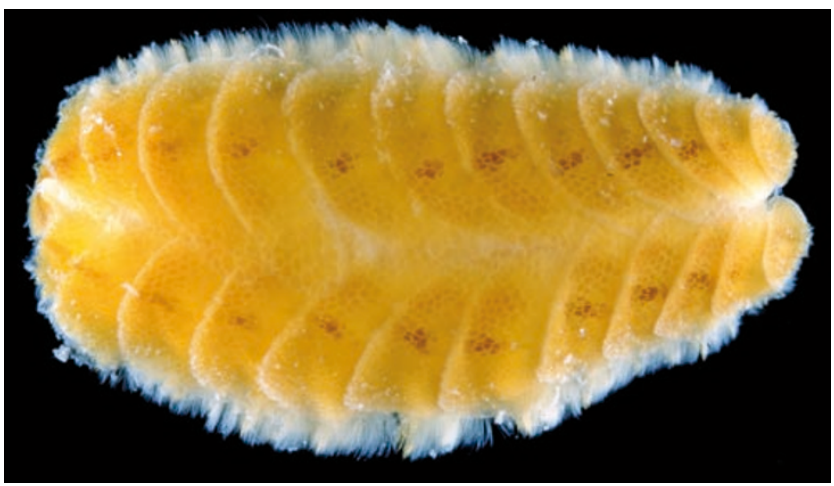

Abb. 1 Iphione ovata (Polynoidae), Dorsalansicht, Vorderende rechts im Bild (Original, T. Wehe)

wirkend vorstellen. Die meisten Arten leben daher auf Hartsubstrat, sei es Felsboden oder Korallen, und weiden den Untergrund ab. Dabei lässt sich oft ein „Stammplatz“ beobachten, häufig als Vertiefung im Untergrund zu erkennen, an den sie nach kurzen Ausflügen der Nahrungssuche zurückkehren. Ganz ähnliche Beobachtungen wurden nun an Iphione gemacht. Wie alle Schuppenwürmer besitzt diese Art serial und paarig angeordnete Rückenschilder, so genannte Elytren, die das Tier komplett bedecken (s. Abb. 1). Diese Rückenschilder sind kräftig ausgebildet und überlappen einander dachziegelartig. Durch eine ebenfalls kräftige und ähnlich den Chitonen ausgebildete Muskulatur leben auch diese Schuppenwürmer tagsüber fest gehaftet am Substrat und wandern nur nachts zur Nahrungsaufnahme ein wenig umher. Wie bei anderen Schuppenwürmern, z. B. bei der im Sediment lebenden Seemaus (Aphrodita aculeata) der Nordsee, kann man auch bei ihnen ein Heben und Senken der Elytren beobachten, wodurch ein Atemwasserstrom erzeugt wird.

Der wabenartige Aufbau der Elytren ist schon früh auf Interesse gestoßen (Kinberg 1856). Es wurden auch Vergleiche zu Pflanzenparenchym gezogen (Pflugfelder 1933). Die genaue Struktur, basierend auf elektronenmikroskopischen Untersuchungen, wurde erst von Storch und Alberti (1995) aufgezeigt. In der Sprache der Ingenieure würde der Aufbau der Elytren wohl als Leichtbauweise bezeichnet werden, wobei Materialersparnis mit gleichzeitiger Festigkeit einhergeht. Auf ultrastruktureller Ebene zeigt sich eine einzigartige Konstruktion des dorsalen Epithels aus einschichtiger Epidermis und Kutikula mit Kammern (Abb. 2).

Der Indo-Westpazifik ist aus zoogeographischer Sicht die Provinz mit der höchsten Biodiversität. Dies gilt fast ausnahmslos für alle marinen Tiergruppen und ist besonders augenfällig bei den farben- und formenprächtigen Korallen, Schnecken oder Rifffischen. In einem Bereich, der sich in etwa von der Malaiischen Halbinsel über die indonesische Inselwelt bis hin nach Neuguinea und den Philippinen er-

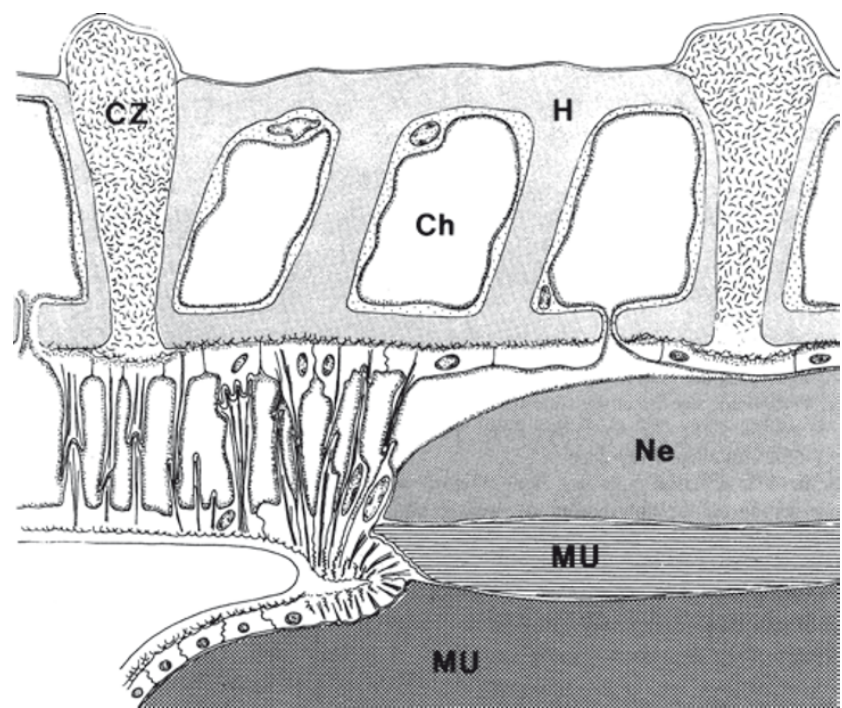

Abb. 2 Schematische Darstellung eines senkrechten Schnittes durch ein Elytron von Iphione muricata. Ch Kammer, CZ Kutikula der Verbindungszone, $H$ homogene Kutikula, $M U$ Muskulatur, $N e$ Nerv (aus Storch und Alberti 1995)

streckt, ist die Artenvielfalt am höchsten. Auch Borstenwürmer bilden hier keine Ausnahme. In den 1980er-Jahren entstanden mehrere systematisch-faunistische Arbeiten zur Polychaetenfauna der Philippinen, in denen drei neue Arten beschrieben und weitere Arten dokumentiert wurden (Rosito 1980; Hartmann-Schröder 1981; Storch und Rosito 1981; Rosito 1983; Hartmann-Schröder 1984; Rosito und Gualberto 1984). Die gesammelten Arten und die Ergebnisse dieser Arbeiten resultieren aus Kursen und Feldstudien die von V. Storch als Gastprofessor im Rahmen einer Kooperation zwischen der Universität Heidelberg und der Universität San Carlos, Cebu City sowie dem Southeast Asian Fisheries Developmental Center SEAFDEC in Tigbauan (Iloilo) durchgeführt wurden. Interessanterweise fand sich unter den untersuchten Borstenwürmern eine große (bis zu $60 \mathrm{~cm}$ Länge) röhrenbauende Art, Loimia medusa, in deren Röhre eine kleine Krabbe, Aphanodactylus sibogae, gefunden wurde, die bis dato nur von einem einzigen Fundort in Indonesien bekannt war. Die Krabbe gehört in die Verwandtschaft der so genannten Muschelwächter, deren Vertreter, wie der Name andeutet, als in Muscheln lebend bekannt sind. Diese Assoziation ist insofern ungewöhnlich, als der Mitbewohner in diesem Fall nicht der Borstenwurm sondern die Krabbe ist. In der Nordsee finden wir eine Assoziation mit umgekehrten Vorzeichen. Die erwachsenen Tiere des großen Einsiedlerkrebses, Pagurus bernhardus, leben in Gehäusen einer der größten Schnecken der Nordsee, dem Wellhorn, Buccinum undatum. Der Krebs reicht mit seinem Hinterleib nicht bis in die hinteren Windungen des Schneckengehäuses. Dort aber findet man regelmäßig einen Nereididen als Mitbewohner, den durchaus $10 \mathrm{~cm}$ erreichenden 
Nereis fucata. Sobald der Krebs seine Beute isst, kommt der Wurm über den Körper des Krebses herausgekrochen und wagt sich bis unmittelbar zu dessen Mundwerkzeugen vor, um am Mahl teilzunehmen.

\subsection{Morphologische Untersuchungen}

Nach moderner Auffassung versteht man unter Biodiversität mehr als nur Artenvielfalt. Biodiversität beinhaltet auch supra- und subspezifische Ebenen, bis hin zur genetischen Vielfalt. So finden wir auch auf der makroskopischen und ultrastrukturellen Ebene eine Vielzahl unterschiedlich ausgeprägter Strukturen: von Organen, Geweben, Zellen bis hin zu Organellen.

Die Untersuchung der morphologischen Vielfalt der Borstenwürmer bildet den Schwerpunkt der wissenschaftlichen Arbeiten von Volker Storch. Bereits mit der minutiösen Darstellung des Muskelsystems von Iphione muricata (Storch 1967b) wird die weitere Ausrichtung der Forschung über die makroskopische Morphologie und Histologie hin zur Ultrastruktur deutlich.

Die Muskulatur der Annelida, damit auch der Borstenwürmer, wird in Lehrbüchern zumeist sehr vereinfacht dargestellt. Der Student erfährt von einem Hautmuskelschlauch mit äußerer Ring- und innerer Längsmuskulatur, zuweilen von dorsoventral verlaufender Muskulatur. Wie viel komplizierter die Verhältnisse im Speziellen sind, wurde beispielhaft von Storch $(1968,1969)$ dargelegt. Nach heutigem Stand der Systematik wurden Vertreter aus 26 Polychaetenfamilien vergleichend anatomisch untersucht, was knapp einem Drittel aller bekannten Familien entspricht. Insgesamt fanden 70 Arten Berücksichtigung. Zur besseren Vergleichbarkeit der Muskulatur verschiedener Arten wurden standardisierte Blockdiagramme sowie in eine Ebene projizierte Schemata der Muskulatur einer Körperseite eines Segmentes angefertigt. Abbildung 3 zeigt die Verhältnisse bei Aphrodita aculeata. Die Untersuchung der Muskulatur hatte auch den Zweck, zum Verständnis der verwandtschaftlichen Beziehungen innerhalb der vielfältigen Gruppe der Borstenwürmer beizutragen. Grundlage und methodisches Rüstzeug dieser Arbeiten bildeten die von Remane (1956) in aller Ausführlichkeit formulierten Kriterien der Homologisierung. Storch (1968) entwickelte einen ursprünglichen Zentraltypus, die Schuppenwürmer im weiteren Sinne, von dem drei Entwicklungslinien ausgehen. Die Verwandtschaftsverhältnisse der Borstenwürmer zueinander sind bis heute unklar und in ständigem Fluss. Morphologische und molekulare Daten lassen sich nur in einigen Fällen zur Deckung bringen, und Monophylie kann nur für wenige Taxa belegt werden, u. a. für die von Storch (1968) als ursprünglich angesehenen Aphroditidae sensu lato. Auch eine kürzlich erschienene molekulare Phylogenie der
Anneliden mit dem bisher umfangreichsten verwendeten Datensatz an Taxa und molekularen Markern konnte die großverwandtschaftlichen Verhältnisse nicht befriedigend auflösen (Rousset et al. 2007). Dass die Muskulatur als morphologisches Merkmal zur Verwandtschaftsanalyse herangezogen werden kann, hat Storch (1969) am Beispiel von benthonischen Polychaeten zeigen können. Arten aus verschiedenen Familien, die im gleichen Lebensraum - in diesem Falle Weichboden - vorkommen, zeigen deutliche Unterschiede in ihrer Muskulatur. Weitgehende Übereinstimmung herrscht aber mit Arten der gleichen Gattung, die in anderen Lebensräumen vorkommen - ein Hinweis darauf, dass die Muskulatur stärker die phylogenetischen Beziehungen als die Umweltbedingungen widerspiegelt.

\subsection{Ultrastrukturelle Untersuchungen: Integument und Verdauungstrakt}

Es wurde bereits erwähnt, dass Biodiversität sich nicht auf die bloße Anzahl von Arten beschränkt. Die intensive Erforschung der Ultrastruktur von Zellen und Geweben durch die Transmissionselektronenmikroskopie, etwa seit den 1960erJahren, hat zu ganz neuen Erkenntnissen geführt. Strukturen und Vorgänge auf subzellulärer Ebene können anhand experimenteller Ansätze und elektronenmikroskopischer Aufnahmen rekonstruiert und auf diese Weise weite Bereiche der Biologie von Organismen, z. B. Fortpflanzung, Ernährung, Sinneswahrnehmung oder Bewegung, erklärt werden. Einen hervorragenden Überblick über die Ergebnisse der ultrastrukturellen Forschung an Borstenwürmern bieten u.a. Olive (1983a,b), Fischer und Pfannenstiel (1984), BereiterHahn et al. (1984), Westheide und Hermans (1988), Harrison und Gardiner (1992), Rouse (1999) sowie Bartolomaeus und Purschke (2005).

Das Integument der Polychaeten wurde von Storch und Welsch (1970, 1972a), Welsch et al. (1984) und Storch (1988) intensiv untersucht. Es besteht aus einer einschichtigen Epidermis, deren Zellen einer Basallamina aufsitzen und apikal von einer Kutikula bedeckt werden. Darunter findet sich Ring- und Längsmuskulatur. Die einzelnen Bestandteile sind je nach Art und Körperabschnitt bzw. Körperanhang unterschiedlich ausgebildet. Besonders auffällig im elektronenmikroskopischen Bild - weil zumeist sehr regelmäßig angeordnet - sind die Kollagenfibrillen der Kutikula. Die Fibrillen sind dabei in etwa rechtwinklig gegeneinander versetzten Lagen angeordnet, wobei die Ausrichtung der Fibrillen jeweils diagonal zur Längsrichtung der Körperachse verläuft (Abb. 4). Auf diese Weise wird eine feste, aber gleichzeitig auch sehr dehnbare Textur geschaffen, deren wichtigsten Aufgaben es sind dem Körper Stabilität zu verleihen - der Hautmuskelschlauch bildet zusammen mit der flüssigkeitsgefüllten Leibeshöhle ein Hydroskelett -, Schutz zu gewährleisten, Kommuni- 
Abb. 3 Schematische Darstellung der Muskulatur von Aphrodita aculeata (Aphroditidae) (aus Storch 1968)

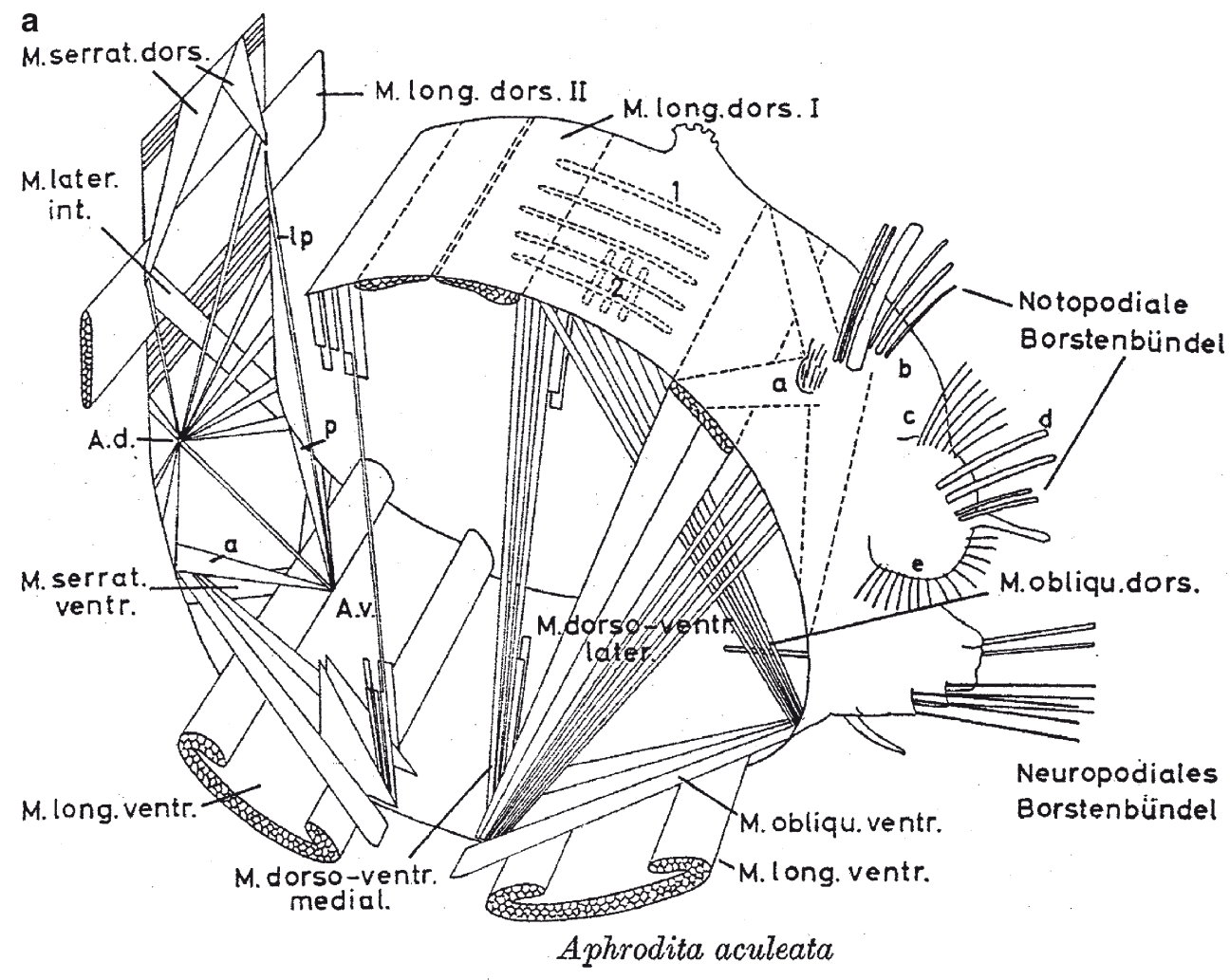

b

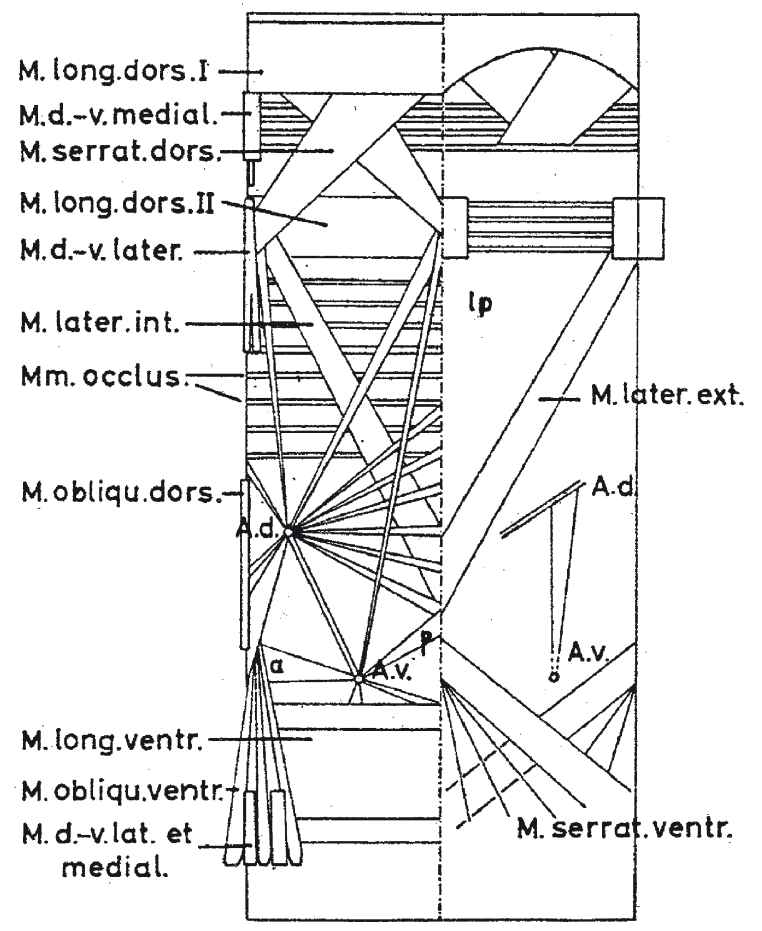

kation mit der Umwelt und die Absorption von Nährstoffen zu ermöglichen. Die Kutikula wird daher vielfach zur Oberfläche hin durchbrochen von Cilien, Mikrovilli oder Ausführgängen von Drüsenzellen bzw. Schleimzellen der Epidermis. Drüsenzellen sind in großer Zahl vorhanden und zeigen bei der Untersuchung im Elektronenmikroskop ebenfalls ein charakteristisches Bild (Abb. 5). In der Epidermis finden sich weiterhin Sinneszellen, Pigment- und Stützzellen sowie die borstenbildenden Chaetoblasten. Eine im Querschnitt getroffene Borste ist nicht nur schön anzusehen (Abb. 6), sondern offenbart auch das Prinzip ihrer Bildung. Die Chitinborste ist von hohlen Röhren 


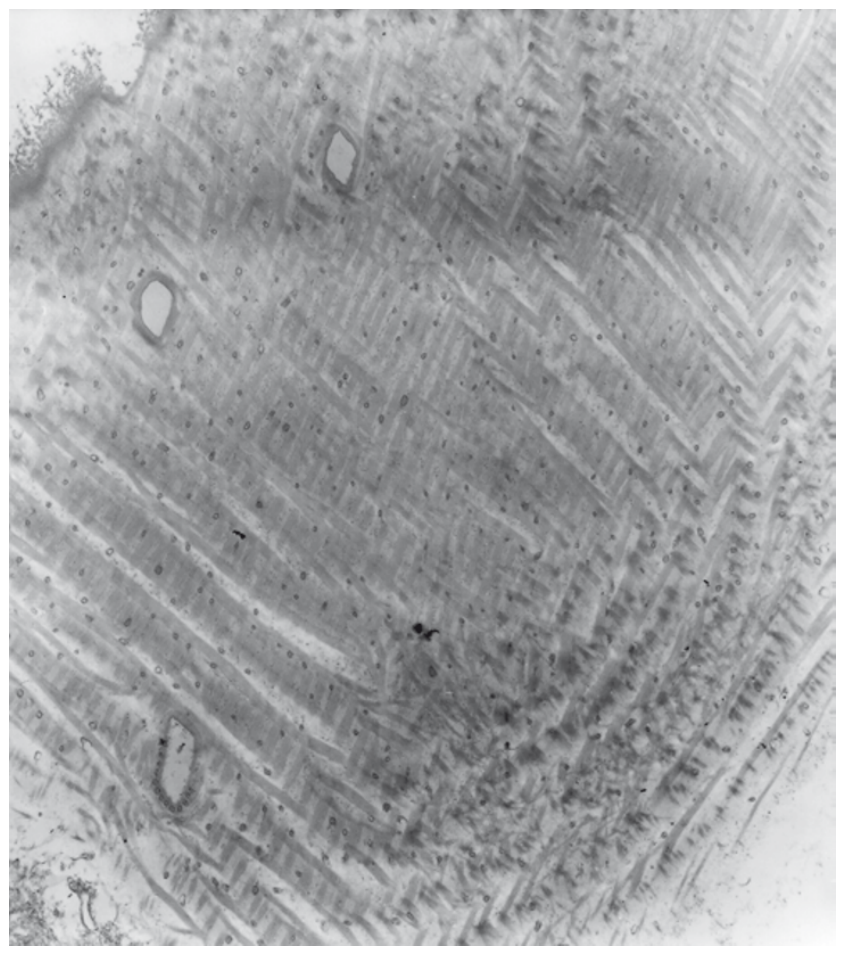

Abb. 4 Kollagenfibrillen der Kutikula von Hypania invalida (Ampharetidae), beachte rechtwinklige Anordnung der Fibrillen, TEM, 5000x (Original, P. Kremer)

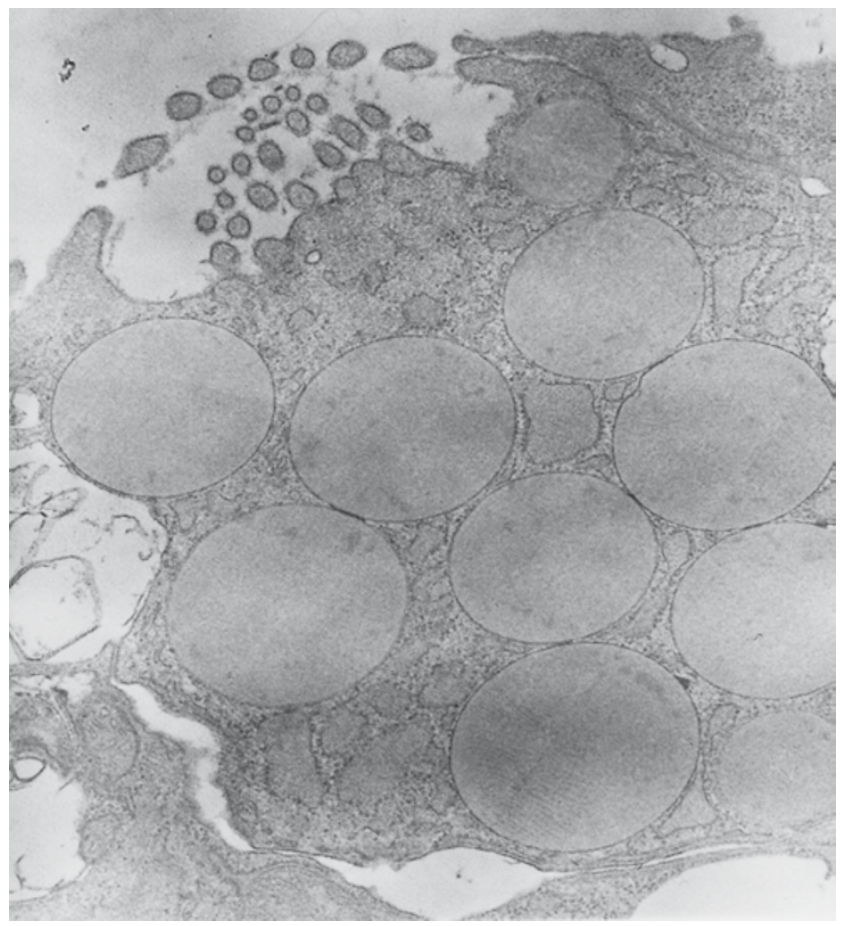

Abb. 5 Sekretvesikel einer epidermalen Drüsenzelle von Hypania invalida, apikaler Pol links oben im Bild, TEM, 20.000x (Original, P. Kremer)

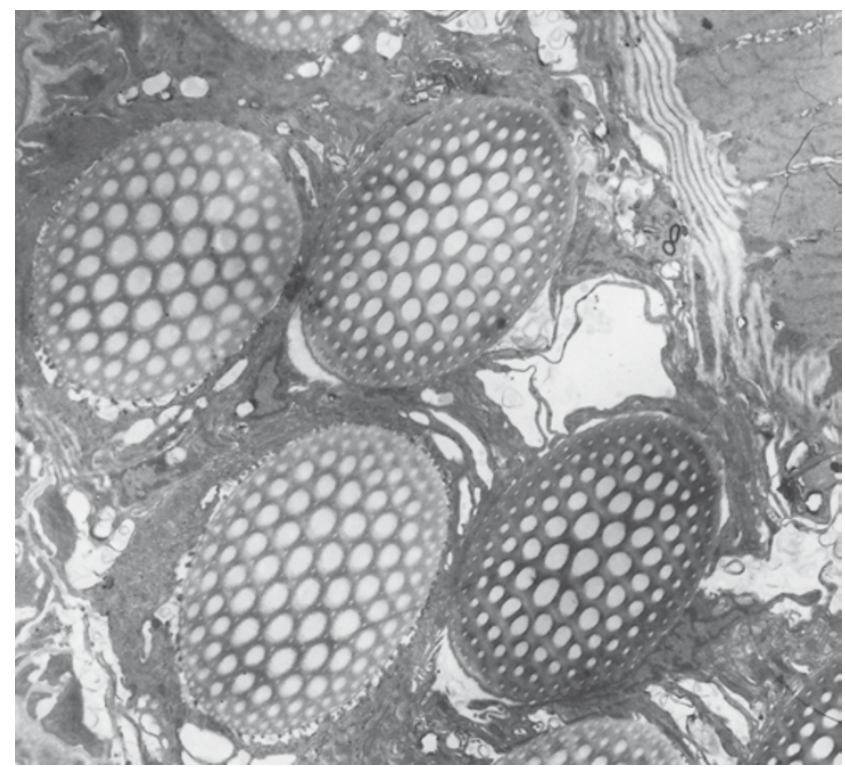

Abb. 6 Quergetroffene Borsten im Integument von Hypania invalida. Das Lochmuster, tatsächlich handelt sich um Röhren, entsteht bei der Bildung der Borsten durch Mikrovilli, TEM, 2000x (Original, P. Kremer)

durchzogen, die dort entstehen, wo am apikalen Pol des Chaetoblasten bei der Bildung des Chitins die Mikrovilli standen.

Das Integument und das Epithel des Verdauungstraktes wurden auch histochemisch untersucht (Storch und Welsch 1972b; Welsch und Storch 1970, 1986). Die Beschaffenheit des Integuments kann die Anforderungen der Umwelt an den Organismus widerspiegeln. Dies zeigen die Ergebnisse zu den atmosphärische Luft atmenden Borstenwürmern von Sumatra (Storch und Welsch 1972b). Diese Nereididen leben in Röhren in Mangroveböden, die nur einmal im Monat bei Springtide vom Wasser überspült werden. Es zeigte sich, dass der Enzymgehalt des Integuments deutlich geringer ist als der typischer mariner Arten. Offensichtlich ist bei diesen Arten die metabolische Aktivität der Epidermis geringer.

\subsection{Ultrastrukturelle Untersuchungen: Sinnesorgane und Körperanhänge}

Eines der wesentlichen Ziele der Systematik ist die Aufklärung verwandtschaftlicher Beziehungen von Arten und höheren Taxa zueinander und die Rekonstruktion der Stammesgeschichte. Die moderne Systematik bedient sich hierbei zahlreicher computergestützter Rechenmodelle und molekularer Methoden bzw. Merkmale. Dabei sollen, wenn möglich, monophyletische bzw. natürliche Verwandtschaftsgruppen identifiziert werden, d.h. Gruppen von Arten, die sich alle auf einen gemeinsamen Ahnen zurückführen las- 
sen. Wichtig in diesem Zusammenhang ist das Erkennen von Synapomorphien, Merkmalen, die nur den Mitgliedern eines Monophylums gemein sind. Solche Charaktere fehlen weitgehend innerhalb der Polychaeta. Ein Merkmal, welches in diesem Zusammenhang diskutiert wird, ist das Nuchalorgan, das bei vielen, aber nicht allen Borstenwürmern nachgewiesen ist. Es handelt sich um ein Chemorezeptororgan am Prostomium (,Kopf') der Würmer. Es ist sehr unterschiedlich gestaltet: von unscheinbaren bewimperten Gruben bis hin zu einem großen Kopfanhang, der Karunkel, der Feuerwürmer (Amphinomidae). Storch und Welsch (1969) haben den Feinbau der Karunkel des Amphinomiden Eurythoë complanata untersucht. Dieses Nuchalorgan ist aus verschiedenen Typen von Sinnes- und weiteren Zellen aufgebaut und stellt damit ein zusammengesetztes Sinnesorgan dar.

Polychaeten besitzen zahlreiche Körperfortsätze: Palpen, Antennen oder Tentakel am Prostomium und darauf folgenden Segmenten, Cirren an den Parapodien entlang des Körpers. Diese Fortsätze werden vielfach als Tastorgane eingesetzt, dementsprechend konnte hier eine Vielzahl von Rezeptorneuronen bei verschiedenen Arten nachgewiesen werden (Storch 1972). Die Anzahl an sensorischen Zellen kann sehr hoch sein, so wurden in einem Dorsalcirrus von Harmothoë über 1000 gefunden. Storch (1973) hat die Sinneszellen der Polychaeten vergleichend mit denen anderer Wirbelloser dargestellt. Auch bei Wirbellosen finden sich generell die drei Exterorezeptortypen: primäre Sinneszelle, freie Nervenendigung, sekundäre Sinneszelle. Bei Borstenwürmern ist der erste Typus weit verbreitet. Eine zusammenfassende Darstellung zur Kenntnis der Rezeptorzellen von Borstenwürmern findet sich bei Storch und SchlötzerSchrehardt (1988).

Neben den genannten Körperanhängen besitzen viele Borstenwürmer Kiemen, welche oft am Vorderkörper sitzen, aber auch entlang der Rückenlinie oder an den Parapodien zu finden sind. Besonders groß und auffällig sind sie bei in Röhren lebenden Formen entwickelt. Storch und Alberti (1978) und Storch und Gaill (1986) haben die Ultrastruktur von neun häufig im Küstenbereich vorkommenden Arten sowie der an hydrothermalen Tiefseequellen in hohen Dichten vorkommenden Alvinella pompejana (Alvinellidae) untersucht. Sie konnten zeigen, dass die Kiemen ausschließlich respiratorische Funktion haben und nicht dem Ionenaustausch oder anderen Transportprozessen durch das Epithel dienen. Allerdings ist der Gasaustausch über die Kiemen unterschiedlich bedeutend in den verschiedenen Arten, was unter anderem durch die Distanz der Blutgefäße zum umgebenden Meerwasser deutlich wird. Eigene Untersuchungen (Kremer und Wehe, in Vorbereitung) wurden hierzu an Hypania invalida (Ampharetidae) aus dem Rhein durchgeführt, dem einzigen Süßwasserpolychaeten in Deutschland.

\section{Schlussfolgerungen}

Der vorliegende Aufsatz kann nur einige wenige Aspekte zur Biodiversität der Borstenwürmer anreissen. Es war die Absicht dies anhand des wissenschaftlichen Beitrags von V. Storch zur Kenntnis der Polychaeta zu tun. Der Autor selbst hat sich intensiv mit der Diversität der Borstenwürmer, insbesondere der Schuppenwürmer des Roten Meeres und den weiteren Meeren um die Arabische Halbinsel, beschäftigt. Fast 800 Arten sind aus der ganzen Region bekannt, mehr als 560 allein aus dem Roten Meer (Wehe und Fiege 2002). Die Revision der Schuppenwürmer aus den Familien der Polynoidae und Sigalionidae resultierte unter anderem in der Beschreibung einer neuen Gattung und neun neuer Arten (Wehe 2006, 2007); nur ein Beispiel unter vielen für unseren immer noch geringen Kenntnisstand vor allem tropischer Gewässer. V. Storch, D. Fiege und T. Wehe haben die Geschichte der Erforschung und die eigenen Beiträge über Borstenwürmer des Vorderen Orients zusammengefasst (Wehe et al. 2006).

2007 hatten M. Reuscher, P. Kremer und T. Wehe die Gelegenheit, im Golf von Akaba weitere Sammlungen durchzuführen. Die Bearbeitung dieser Proben ist gegenwärtig noch im Gange. Bereits jetzt sind aber schon einige Neunachweise identifiziert worden. Unter den Ampharetidae wurde eine neue Art gefunden. In Hochachtung seiner herausragenden wissenschaftlichen Leistung und seiner Verbundenheit mit der Tiergruppe der Borstenwürmer, widmen M. Reuscher und T. Wehe diese neue Art Herrn Prof. Dr. Dr. h.c. Volker Storch.

\section{Description of the new species}

Family Ampharetidae, Malmgren, 1866

Subfamily Ampharetinae, Chamberlin, 1919

Genus Samytha, Malmgren, 1866

Type species: Sabellides sexcirrata, Sars, 1856

Generic diagnosis Prostomium without glandular ridges. Buccal tentacles smooth. Three pairs of smooth branchiae. No paleae. Thorax with 17 chaetiger, 14 unciniger. No notopodial cirri.

\section{Samytha storchi Reuscher \& Wehe nov. spec. (Fig. 7)}

Specimens examined Holotype, complete specimen (SMF 18125), Red Sea, Gulf of Aqaba, Jordan, Aqaba, South of Marine Science Station, reef in front of tourist beach, coral rubble; 5-15 m, leg. M. Reuscher, P. Kremer, Y. Ahmed, 16 December 2007.

Description Holotype complete specimen, length $11 \mathrm{~mm}$, width $1.5 \mathrm{~mm}$ on widest part of thorax, 30 segments, fema- 
a

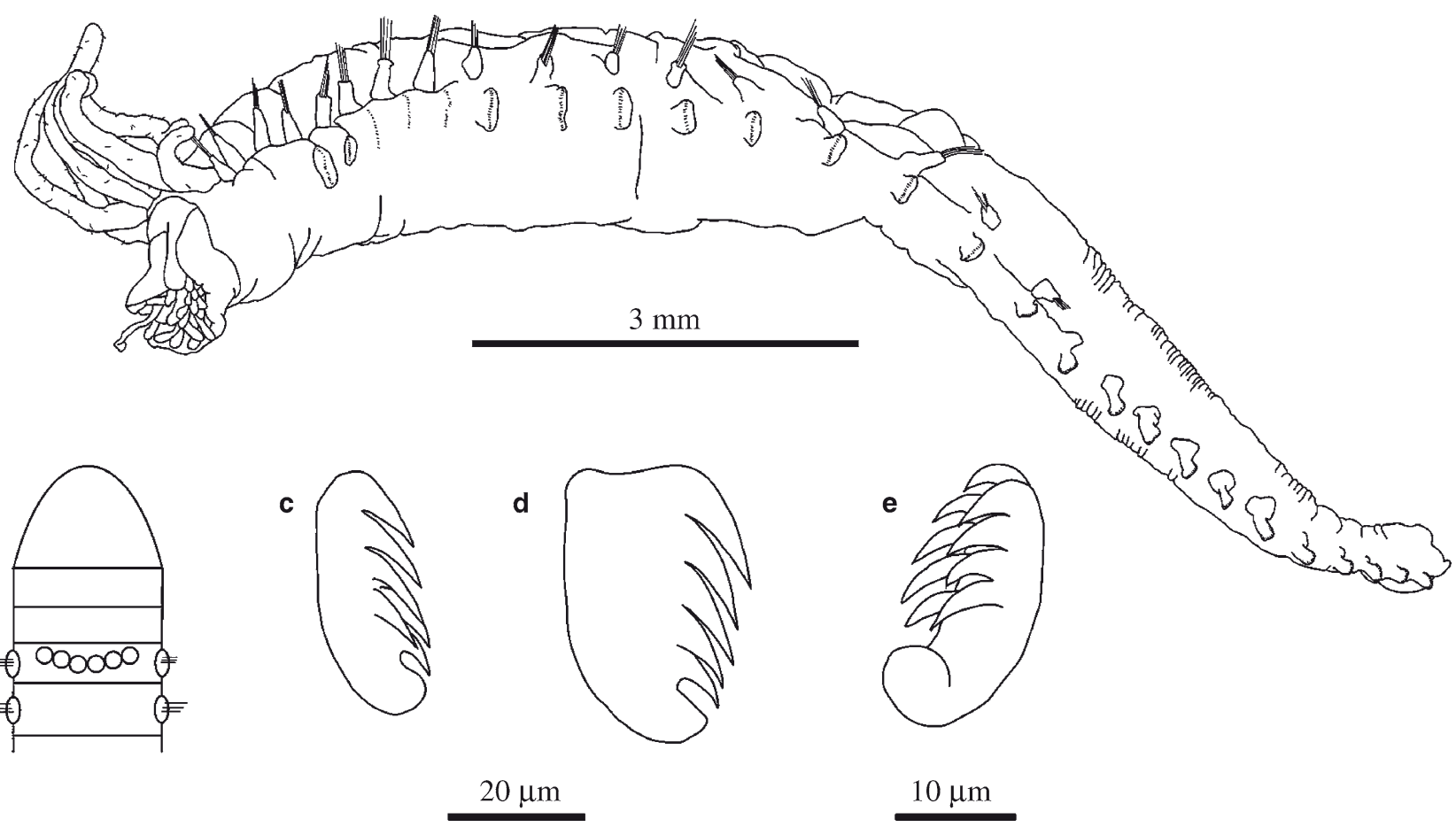

Fig. 7 a Samytha storchi Reuscher and Wehe nov. spec., holotype, complete specimen, lateral view; b schematic representation of the arrangement of the branchiae on first chaetiger; $\mathbf{c}, \mathbf{d}$ thoracic uncini;

e abdominal uncini

le. Body slender, integument in part transparent, eggs visible through body wall. Body from about unciniger 6 and 7 gradually tapering towards pygidium (Fig. 7a). Prostomium trilobed, without glandular ridges or eye-spots. Buccal tentacles mostly retracted, smooth where visible, without grove. Three pairs of cirriform branchiae without furrows, with minute annuli; bases of all branchiae broad and fused to each other. All three pairs of branchiae in transverse row on chaetiger 1 (segment III), arrangement of bases crescentshaped with outer pair of branchiae situated anteriorly and middle pair of branchiae posteriorly (Fig. 7b). No chaetae arising from segment II. 17 thoracic chaetiger. Notopodia of all chaetiger clearly developed. 14 thoracic unciniger, first three chaetiger without tori and uncini. Notopodia without cirri. Segment borders only visible on thorax in ventral view. Abdomen short, only about one third of entire body length, comprising 11 segments. Abdomen with distinct ventral grove. Glandular pads above abdominal neuropodia. Pygidium with two very short anal cirri. Capillary chaetae bilimbate under light microscope. Thoracic and abdominal uncini of different shape; thoracic uncini with 3 teeth in vertical row, followed by two teeth in horizontal order and a single tooth above basal prow, or five teeth in one vertical row above basal prow (Fig. 7c,d); uncini of abdomen with single upper tooth above two vertical rows of four teeth each followed by the basal prow (Fig. 7e).
Remarks This new species can easily be distinguished from other species within the genus Samytha by the six branchiae, the bases of which are all fused and located on the first chaetiger. In other species there is a gap between the left and right group of branchiae. Thoracic uncini with teeth arranged in the pattern 1-1-1-2-1 have not been described for other species of Samytha. The number of $11 \mathrm{ab}-$ dominal segments is the lowest mentioned in the literature and the specimen is sexually mature. In contrast to the most similar species $S$. californiensis Hartman 1969, the pygidium of the newly described species is not conspicuously crenulated.

Etymology The species is named for Volker Storch, a much valued teacher and zoologist, who started his scientific career working on polychaetes from the Red Sea, on the occasion of his 65th birthday and in honour of his tremendous contribution to our zoological knowledge.

Acknowledgements The species was collected in 2007 during one of two sampling trips to the Marine Science Station in Aqaba. Visits to the Station were generously funded by the DAAD (German Academic Exchange Service) project „Establishment of a Middle Eastern Biodiversity Research, Training and Conservation Network". The support of the polychaete research activities by the Ichthyology Section at the Research Institute Senckenberg, Frankfurt a. M. (Dr. Friedhelm Krupp, Mrs. Nadia Manasfi) was indispensible. We are also indebted to the counterparts at the Marine Science Station, namely Dr. Fuad AlHorani and the late Yousef Ahmed. 


\section{Literatur}

Bartolomaeus T, Purschke G (Hrsg) (2005) Morphology, molecules, evolution and phylogeny in Polychaeta and related taxa. Developments in Hydrobiology 179, Springer, Dordrecht, 397 p

Bereiter-Hahn J, Matoltsy AG, Sylvia Richards K (Hrsg) (1984) Biology of the Integument 1. Invertebrates. Springer Verlag, Berlin, $841 \mathrm{p}$

Bouchet P (2006) 2. The magnitude of marine biodiversity. In Duarte CM (Hrsg) The Exploration of Marine Biodiversity. Scientific and Technological Challenges, Fundación BBVA, Bilbao, 31-62

Chamberlin RV (1919) The Annelida Polychaeta. Mem. Mus. Comparative Zoölogy at Harvard College 48:1-514

Coleman N, Gason ASH, Poore GCB (1997) High species richness in the shallow marine waters of south-east Australia. Marine Ecology Progress Series 54:17-26

Fischer A, Pfannenstiel H-D (Hrsg) (1984) Polychaete reproduction. Progress in comparative reproductive biology. Fortschritte der Zoologie 29, Stuttgart, Gustav Fischer Verlag, Stuttgart, 341 p

Grassle JF, Maciolek NJ (1992) Deep-sea species richness: regional and local diversity esimates from quantitative bottom samples. Am Nat 139(2):313-341

Grube E (1868) Beschreibungen einiger von Georg Ritter von Frauenfeld gesammelter Anneliden und Gephyreen des rothen Meeres. Verhandlungen der kaiserlich-königlichen zoologisch-botanischen Gesellschaft in Wien 18:629-650

Grube E (1869) Beschreibungen neuer oder weniger bekannter von Hrn. Ehrenberg gesammelter Anneliden des rothen Meeres. Monatsberichte der Königlich Preussischen Akademie der Wissenschaften zu Berlin aus dem Jahre 1869:484-521

Harrison FW, Gardiner SL (Hrsg) (1992) Annelida. Microscopic Anatomy of Invertebrates 7. New York, Willey-Liss, New York, 418 p

Hartman O (1969) Atlas of the sedentariate polychaetous annelids from California. Allan Hancock Foundation, University of Southern California, Los Angeles, $812 \mathrm{pp}$

Hartmann-Schröder G (1981) Hololepidella alba, a new polychaete species associated with Acanthaster planci. The Philippine Scientist 18:10-14

Hartmann-Schröder G (1983) 5.4 The ragworm Nereis diversicolor. In: Dankers N, Kühl H, Wolff WJ (eds) Invertebrates of the Wadden Sea. In: Wolff WJ (Hrsg) Ecology of the Wadden Sea Vol. 1. A.A. Balkema, Rotterdam, pp 4/113-4/114

Hartmann-Schröder G (1984) Zwei neue kommensalische Polychaeten der Gattung Hololepidella Willey (Polynoidae) von den Philippinen. Mitt Hamb Zool Mus 81:63-70

Hartmann-Schröder G (1993) 1. Klasse Polychaeta, Vielborster, S. 288-374. In: Gruner HE (Hrsg.) Lehrbuch der Speziellen Zoologie. Begründet von A. Kaestner, 5. Aufl. Gustav Fischer Verlag, Jena, $608 \mathrm{~S}$

Hutchings PA, Fauchald K (2000) Class Polychaeta, S. 1-3. In: Beesley PL, Ross GJB, Glasby CJ (Hrsg) Polychaetes \& Allies: The Southern Synthesis. Fauna of Australia Vol. 4A Polychaeta, Myzostomida, Pogonophora, Echiura, Sipunculida. CSIRO Publishing, Melbourne, xii $465 \mathrm{~S}$

Kinberg JGH (1856) Nya slägten och arter af Annelider. Öfversigt af Kongliga Vetenskaps-Akademiens Förhandlingar 12(9\&10): $381-388$

Kundy M (1990) Wattwurm (Arenicola marina), S. 121-128. In Landesinstitut Schleswig-Holstein für Praxis und Theorie der Schule und Landesamt für den Nationalpark Schleswig-Holsteinisches Wattenmeer (Hrsg) Tiere im Wattenmeer. Verlag Schmid und Klaunig, Kiel, $251 \mathrm{~S}$

Lambshead PJD, Boucher G (2003) Marine nematode deep-sea biodiversity-Hyperdiverse or hype? J Biogeogr 30:475-485

Malmgren AJ (1866) Nordiska hafs-annulater. Öfversigt af Kongliga Vetenskaps-Akademiens Förhandlingar 22:355-410
Martin D, Britayev TA (1998) Symbiotic polychaetes: review of known species. Oceanography and Marine Biology. An Annual Review 36:217-340

Olive PJW (1983a) 15. Annelida-Polychaeta. In: Adiyodi KG, Adiyodi RG (Hrsg) Reproductive Biology of Invertebrates. Volume I: Oogenesis, Oviposition, and Oosorption. John Wiley \& Sons Ltd., Chichester, pp 357-422

Olive PJW (1983b) 16. Annelida-Polychaeta. In: Adiyodi KG, Adiyodi RG (Hrsg) Reproductive Biology of Invertebrates. Volume II: Spermatogenesis and Sperm Function. John Wiley \& Sons Ltd., Chichester, pp 321-342

Pflugfelder O (1933) Zur Histologie der Elytren der Aphroditiden. Zeit wiss Zool 143:497-537

Remane A (1956) Die Grundlagen des natürlichen Systems, der Vergleichenden Anatomie und der Phylogenetik. 364 S. Akademische Verlagsgesellschaft Geest \& Portig K.-G., Leipzig

Remane A, Schulz E (1964) Die Strandzonen des Roten Meeres und ihrer Tierwelt. Kieler Meeresforschungen 20 (Sonderheft):5-17

Rosito R (1980) Polychaeta from shallow waters off Mactan, Cebu. Part I. The Philippine Scientist 17:1-35

Rosito R (1983) Polychaetes from shallow waters off Mactan, Cebu. Part II. The Philippine Scientist 20:11-38

Rosito R, Gualberto EP (1984) New records of pelagic polychaetous annelids from Mactan Island, Cebu, Central Philippines. The Philippine Scientist 21:1-10

Rouse GW (1999) 3. Polychaeta, including Pogonophora and Myzostomida. In: Adiyodi KG, Adiyodi RG (Hrsg) Reproductive Biology of Invertebrates 9B: Progress in male gamete ultrastructure and phylogeny, New Dehli, Oxford \& IBH Publishing CO. PVT. Ltd., pp 81-124

Rousset V, Pleijel F, Rouse GW, Erséus C, Siddall ME (2007) A molecular phylogeny of annelids. Cladistics 23:41-63

Sanders HL (1968) Marine benthic diversity: a comparative study. Am Nat 102(925):243-321

Sars M (1856) Nye Annelider. Fauna littoralis Norvegiae 2. 24 S. Fredrik D. Beyer, Bergen

Savigny JC (1818) In: Lamarck JBD: Histoire naturelle des Animaux sans vertèbres. Vol. 5. 612 S. Paris, Déterville et Verdière

Savigny JC (1822) Systèmes de diverses classes d'animaux sans vertèbres. Système des annélides, principalement de celles des côtes de l'Égypte et de la Syrie, offrant les caractères tant distinctifs que naturels des ordres, familles et genres, avec la description des espèces. $128 \mathrm{~S}$. (Sonderdruck aus Description de l'Égypte)

Snelgrove PVR, Blackburn TH, Hutchings P, Alongi D, Grassle JF, Hummel H, King G, Koike I, Lambshead PJD, Ramsing NB, Solis-Weiss V, Freckman DW (1997) The importance of marine sediment biodiversity in ecosystem processess. Ambio 26(8): $578-583$

Storch V (1966) Drei neue Polychaeten aus dem Litoral des Roten Meeres. Kieler Meeresforschungen 22:171-175

Storch V (1967a) Neue Polychaeten aus der Sandfauna des Roten Meeres. Zoologischer Anzeiger 178:102-110

Storch V (1967b) Iphione muricata (Savigny), ein den Chitonen ähnlicher Lebensformtyp unter den Polychaeten. Kieler Meeresforschungen 23(2):148-155

Storch V (1968) Zur vergleichenden Anatomie der segmentalen Muskelsysteme und zur Verwandtschaft der Polychaeten-Familien. Z Morph Tiere 63:251-342

Storch V (1969) Muskelsysteme benthonischer Polychaeten als Kriterium für die Verwandtschaftsforschung. Kieler Meeresforsch. 25(2):331-335

Storch V (1971) Meeresborstenwürmer. Die Neue Brehm-Bücherei 442. Ziemsenverlag, Wittenberg Lutherstadt, $80 \mathrm{~S}$

Storch V (1972) Elektronenmikroskopische Untersuchungen an Rezeptoren von Anneliden (Polychaeta, Oligochaeta). Z mikrosk.anat Forsch 85(1)55-84 
Storch V (1973) Vergleichende elektronenmikroskopische Untersuchungen über Receptoren von Wirbellosen (Nemertinen, Turbellarien, Molluscen, Anneliden, Aschelminthen). Comparative electron microscopical investigations on receptors of invertebrates (Nemrtini, Turbellaria, Mollusca, Annelida, Aschelminthes). Verhandlungen der Deutschen Zoologischen Gesellschaft. 66. Jahresversammlung vom 10.-15.9. 1972 in Mainz, S 61-65

Storch V (1988) I. Integument. In Westheide W, Hermans CO (Hrsg) The ultrastructure of Polychaeta. Microfauna Marina 4:13-36. Akademie der Wissenschaften, Mainz und Gustav Fischer Verlag, Stuttgart

Storch V, Alberti G (1978) Ultrastructural observations on the gills of polychaetes. Helgol Wiss Meeresunters 31:169-179

Storch V, Alberti G (1995) Elytra of Iphione muricata (Savigny) a reinterpretation of its architecture based on TEM. Mitt hamb zool Mus Inst 92:55-60

Storch V, Gaill F (1986) Ultrastructural observations on feeding appendages and gills of Alvinella pompejana (Annelida, Polychaeta). Helgol Meeresunters 40:309-319

Storch V, Niggemann R (1967) Auf Echinodermen lebende Polychaeten. Kieler Meeresforschungen 23(2):156-164

Storch V, Rosito RM (1981) Polychaetes from interspecific associations found off Cebu. The Philippine Scientist 18:1-9

Storch V, Schlötzer-Schrehardt, U (1988) VIII. Sensory Structures. In Westheide W, Hermans CO (Hrsg) The ultrastructure of Polychaeta. Microfauna Marina 4:13-36. Akademie der Wissenschaften, Mainz und Gustav Fischer Verlag, Stuttgart

Storch V, Wehe T (2007) Biodiversität mariner Organismen: Entstehung - Umfang - Gefährdung. Umweltwiss Schadst Forsch 19(4):213-218

Storch V, Welsch U (1969) Zur Feinstruktur des Nuchalorgans von Eurythoë complanata (Pallas) (Amphinomidae, Polychaeta). Z Zellf 100:411-420

Storch V, Welsch U (1970) Über die Feinstruktur der PolychaetenEpidermis (Annelida). Z Morph Tiere 66:310-322
Storch V, Welsch U (1972a) The ultrastructure of epidermal mucous cells in marine invertebrates (Nemertini, Polychaeta, Prosobranchia, Opisthobranchia). Mar Biol 13(2):167-175

Storch V, Welsch U (1972b) Ultrastructure and histochemistry of the integument of air-breathing polychaetes from mangrove swamps of Sumatra. Mar Biol 17:137-144

Storch V, Welsch U (2004) Systematische Zoologie, 6. Aufl., Spektrum Akademischer Verlag, Heidelberg, xvi $853 \mathrm{~S}$

Vine P (1986) Red Sea Invertebrates. Immel Publishing, London, $224 \mathrm{~S}$

Wehe T (2006) Revision of the scale worms (Polychaeta: Aphroditoidea) occurring in the seas surrounding the Arabian Peninsula. Part I: Polynoidae. Fauna of Arabia 22:23-197

Wehe T (2007) Revision of the scale worms (Polychaeta: Aphroditoidea) occurring in the seas surrounding the Arabian Peninsula. Part II: Sigalionidae. Fauna of Arabia 23:41-124

Wehe T, Fiege D (2002) Annotated checklist of the polychaete species of the seas surrounding the Arabian Peninsula: Red Sea, Gulf of Aden, Arabian Sea, Gulf of Oman, Arabian Gulf. Fauna of Arabia 19:7-238

Wehe T, Fiege D, Storch V (2006) Borstenwürmer des Vorderen Orients. Natur und Museum 136(9/10):197-208

Welsch U, Storch V (1970) Histochemical and fine structural observations on the alimentary tract of Aphroditidae and Nephtyidae (Polychaeta Errantia). Mar Biol 6(2):142-147

Welsch U, Storch V (1986) Carbohydrate histochemistry of polychaetes. Zool Anz 217(5/6):289-300

Welsch U, Storch V, Sylvia Richards K (1984) Chapter 17 Epidermal Cells. In Bereiter-Hahn J, Matoltsy AG, Sylvia Richards K (Hrsg) (1984) Biology of the Integument 1. Invertebrates. Springer Verlag, Berlin, pp 269-296

Westheide W, Hermans CO (Hrsg) (1988) The ultrastructure of Polychaeta. Microfauna Marina 4:494. Akademie der Wissenschaften, Mainz und Gustav Fischer Verlag, Stuttgart 